\title{
LRP: a multifunctional scavenger and signaling receptor
}

\author{
Joachim Herz ${ }^{1}$ and Dudley K. Strickland ${ }^{2}$ \\ ${ }^{1}$ Department of Molecular Genetics, Dallas, Texas, USA \\ ${ }^{2}$ Department of Vascular Biology, Holland Laboratory, American Red Cross, Rockville, Maryland, USA \\ Address correspondence to: Joachim Herz, Department of Molecular Genetics, 5323 Harry Hines Boulevard, \\ Dallas, Texas 75390-9046, USA. Phone: (214) 648-5633; Fax: (214) 648-8804; E-mail: herz@usa.redcross.org.
}

J. Clin. Invest. 108:779-784 (2001). DOI:10.1172/JCI200113992.

\section{Introduction}

The LDL receptor-related protein (LRP) is larger than but structurally similar to other members of the LDL receptor gene family, an ancient family of endocytic receptors (1-3). Whereas the LDL receptor, the founding member of this family, appears to act solely in lipoprotein metabolism, the LRP and other members of this family appear to have other distinct functions. In this article, we will focus on the diverse biological roles of the LRP, which include functions in lipid metabolism, and also in the homeostasis of proteinases and proteinase inhibitors, cellular entry of viruses and toxins, activation of lysosomal enzymes, cellular signal transduction, and neurotransmission.

\section{Structural organization of LRP}

LRP, like all members of the LDL receptor gene family, consists of five common structural units shown in Figure 1: (a) ligand-binding (complement) type cysteinerich repeats, (b) epidermal growth factor (EGF) receptor-like cysteine-rich repeats, (c) YWTD domains, (d) a single membrane-spanning segment, and (e) a cytoplasmic tail that harbors between one and three NPxY motifs. Ligand-binding-type repeats in LRP occur in clusters containing between two and eleven individual repeats. Most of the known ligands for LRP (Table 1), for which the binding sites have been mapped, interact with these ligand-binding-type domains (4). These are followed by EGF precursor homology domains, which consist of the two EGF repeats, six YWTD repeats that are arranged in a propeller-like structure (5), and another EGF repeat. Six EGF repeats precede the single membrane-spanning segment. The cytoplasmic tail contains two NPxY motifs that serve as docking sites for the endocytosis machinery and for cytoplasmic adaptor and scaffolding proteins involved in signaling events (6).

\section{Ligand families and subgroups and}

their binding sites on LRP

LRP recognizes at least 30 different ligands (Table 1) that represent several families of proteins. These include lipoproteins, proteinases, proteinaseinhibitor complexes, ECM proteins, bacterial toxins, viruses, and various intracellular proteins. By far the largest group of ligands that are recognized by LRP

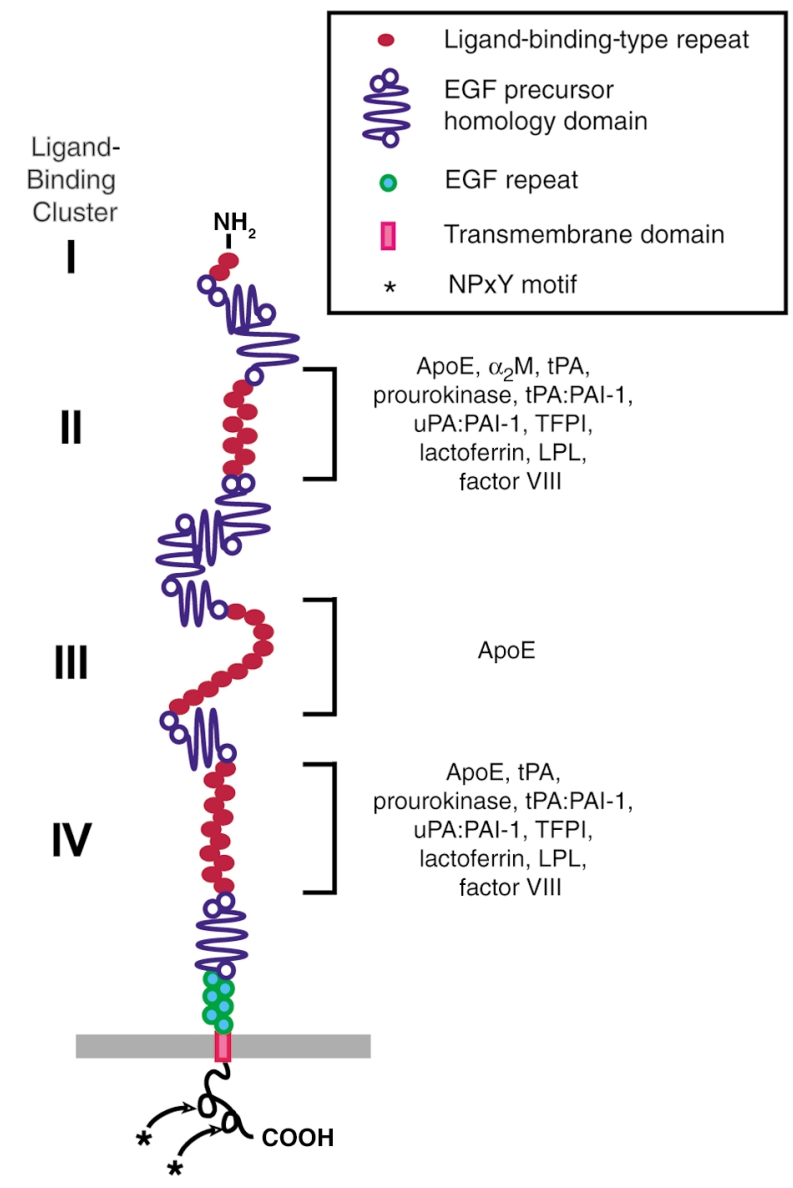

Figure 1

Binding of LRP ligands to the different clusters of ligand-binding repeats. Cysteine-rich ligand-binding repeats (red ovals) in LRP are arranged in four clusters containing 2, 8, 10, and 11 repeats, respectively. Each cluster is followed by 1-4 EGF homology domains (blue), which consist of cysteine-rich EGF repeats (blue circles) and YWTD domains (wavy line). NPXY motifs in the cytoplasmic tail are indicated by the asterisks. No ligand interactions have been mapped to cluster I. Clusters II and IV bind most of the currently mapped known ligands of LRP. Binding of $\alpha_{2} M$ to clusters II and IV has been found by surface plasmon resonance, although cells transfected with minireceptors containing these clusters do not bind and internalize $\alpha_{2} \mathrm{M}$ (8). ApoE was found to bind to clusters II, III, and IV by ligand blotting. LPL, lipoprotein lipase. 
Table 1

Ligands that bind the extracellular domain

$\begin{array}{ll}\begin{array}{l}\text { ApoE } \\ \text { Lipoprotein lipase }\end{array} & \text { Lipoprotein metabolism and transport } \\ \text { Hepatic lipase } & \\ \text { tPA } & \text { Fibrinolysis, signaling function in brain } \\ \text { uPA } & \text { Cell migration, wound healing } \\ \begin{array}{l}\text { Factor IXa } \\ \text { Factor VIIla }\end{array} & \text { Blood coagulation } \\ \text { Factor VIla/TFPI } & \\ \begin{array}{l}\text { MMP-13 } \\ \text { MMP-9 }\end{array} & \text { Angiogenesis, metastasis } \\ \text { Spingolipid activator protein (SAP) }\end{array}$

Pregnancy Zone Protein Pan-proteinase inhibitors, infection $\alpha_{2} \mathrm{M}$

Complement C3

Infection

PAI-1

Regulates tPA/uPA activity

C1 inhibitor

Regulates C1r/C1s activity

Antithrombin III

TFPI

Heparin cofactor II

$\alpha_{1}$-Antitrypsin

Regulates neutrophil elastase

APP

Physiological role unclear

Thrombospondin-1

TGF- $\beta$ activation, matrix cell interactions

Thrombospondin-2

Pseudomonas exotoxin A

Lactoferrin

Antibacterial

Rhinovirus

RAP

Chaperone

HSP-96

Chaperone

HIV-Tat protein

Transcriptional activation tein, and RAP, an endoplasmic reticulum resident protein that functions as a molecular chaperone for LRP and other LDL receptor family members.

A major question that remains unanswered is how LRP can recognize 30 structurally distinct ligands with high affinity. Earlier work with the LDL receptor revealed that the complement-type ligand-binding repeats are responsible for its recognition of ligands; consequently most work has focused on the four clusters of ligand-binding repeats that are present in LRP. Crystallographic and nuclear magnetic resonance studies of individual repeats have revealed that the sequence variability in short loop regions of each repeat results in a unique contour surface and charge density for each repeat (7).

While the locations of the ligand recognition sites for all ligands within LRP are not yet known, two general approaches have been successfully employed to identify the regions responsible for binding a number of ligands. In the first approach (8), LRP "minireceptors" have been prepared by fusing various clusters of ligandbinding repeats to the LRP light-chain and measuring their ability to mediate the cellular internalization of ligands following expression in cells. In the second approach (5), soluble recombinant receptor fragments representing each of the clusters in LRP are tested for their ability to bind various known LRP ligands in vitro.

Together, these studies have yielded some important insights into the ligand recognition properties of LRP. First, it appears that the major ligand-binding sites within LRP are contained in clusters II and IV; thus far, no ligands besides RAP have been identified that bind to cluster I or cluster III (Figure 1). Interestingly, most ligands bind equally well to both clusters II and IV, suggesting a functional duplication within LRP (5). Second, deletion analysis reveals that high-affinity binding of most ligands with LRP requires their interaction with multiple ligand-binding repeats. Some ligands, such as $\alpha_{2} M^{*}$, appear to bind repeats from two or more clusters. These studies reveal that the ability of LRP to bind numerous structurally distinct ligands with high affinity arises from the presence of 31 ligand-binding-type repeats in the molecule, from the unique contour surface and charge distribution for each repeat, and from multiple interactions between both the ligand and the receptor. Some ligands can recognize different combinations of these repeats in a sequential fashion, while others appear to recognize repeats from separate clusters.

\section{Interactions with intracellular adapter} and scaffold proteins

Not only does the extracellular domain of LRP bind a multitude of biologically diverse ligands (Figure 1; Figure 2, left; Table 1), but there are now also an increasing number of cytoplasmic proteins that have been found to interact with the tail of LRP (Figure 2, middle and right; Table 2). A search for such proteins was initiated because it became impossible to reconcile a bewildering spectrum of experimental observations relating to various functions of LRP and other members of the LDL receptor gene family with a simple role as an endocytic receptor or cellular transporter of extracellular ligands. 

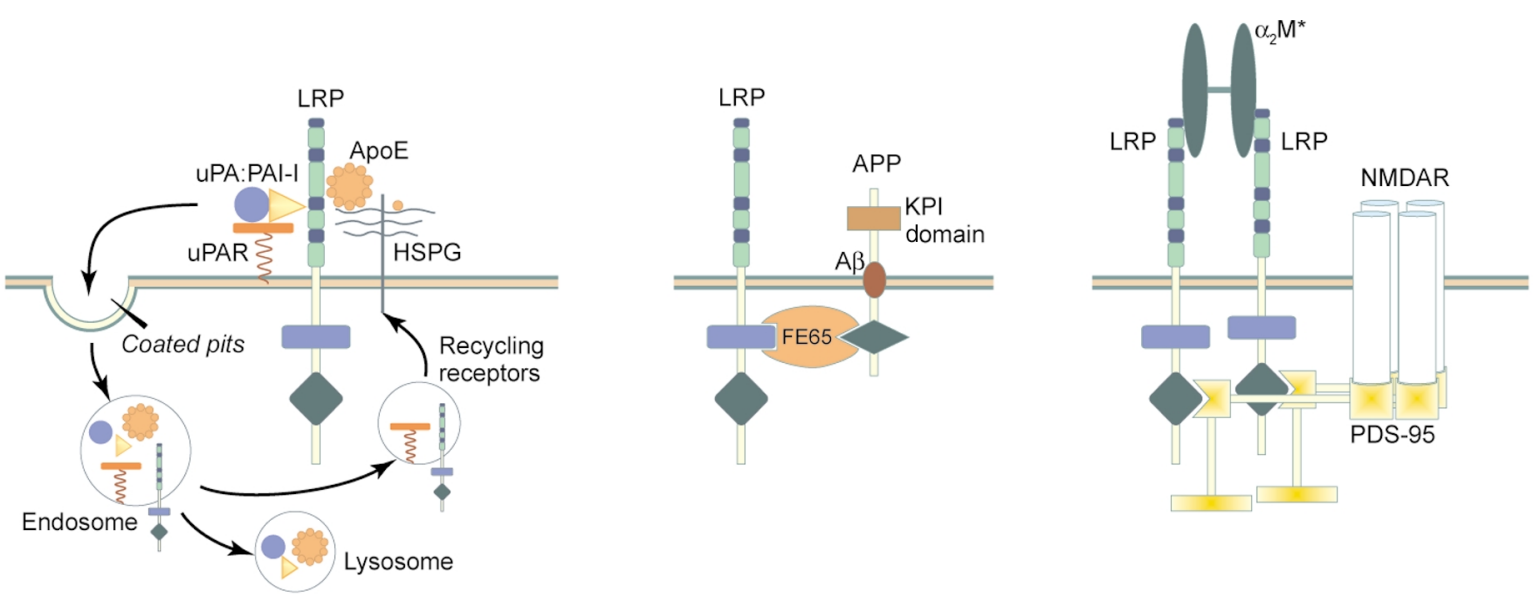

\section{Figure 2}

Extracellular and intracellular associations of LRP with membrane-bound ligands. Left panel: Internalization of GPI-linked or heparan sulfate proteoglycan-linked (HSPG-linked) ligands (examples: uPA:PAl-1, uPA receptor, and apoE-containing lipoproteins). In uPA:PAI-1, PAI-1 binds to LRP and uPA binds to the GPI-linked uPA receptor. ApoE-containing lipoproteins are sequestered on HSPGs, facilitating enrichment with apoE and the interaction with LRP. After internalization and dissociation of the ligands from the receptors in the endosome, receptors recycle to the plasma membrane. Middle panel: Association of LRP with APP mediated by the cytoplasmic scaffolding protein FE65. FE65 can interact via independent PTB domains with the LRP and APP tails. The functional significance of this potential cytoplasmic link for APP processing and/or signaling remains to be shown. Right panel: Dimerization of LRP by $\alpha_{2} M$ and association with NMDA receptors (NMDAR). Dimerization of LRP on cultured neurons induces NMDA receptor-mediated Ca ${ }^{2+}$ influx. The postsynaptic density protein PSD-95 interacts with LRP and NMDA receptors through different interaction domains. How precisely NMDA receptors are activated by LRP dimerization is not known at present.

Initially, the search focused on the tetra-amino acid "NPxY" motif that is present in two copies in the LRP tail. Both motifs can independently function as internalization signals. However, one such motif is sufficient to mediate rapid endocytosis of the LDL receptor (9), suggesting that one or both of these motifs might interact with other proteins in the cytoplasm, in addition to the endocytotic machinery. NPxY motifs had just been shown to interact with cytoplasmic proteins harboring so-called phosphotyrosine-binding (PTB) domains. Furthermore, in an increasing number of cases it became apparent that phosphorylation of the tyrosine residue in these motifs was not required for binding to PTB domains. Two cytoplasmic, PTB domain-containing proteins were originally described that can interact specifically with the NPxY motifs in the LRP tail. One of these proteins, mammalian Disabled-1 (Dab1), specifically binds the second NPxY motif in the LRP tail and also to equivalent sequences in other LDL receptor family members, and it plays a critical role in the transmission of a positional signal to migrating neurons during brain development (10). The second such protein, the scaffold protein FE65, contains two PTB domains and one WW domain by which it can specifically interact with LRP (6), with the amyloid precursor protein (APP) $(11,12)$, and with members of the Ena/VASP family of actin-binding proteins (13). Two FE65-like proteins also exist in mammalian species. Both interact via one of their PTB domains with $\operatorname{APP}(14,15)$. Whether the other PTB domain in FE65L1 and FE65L2 interacts with LRP has not been tested, but it seems likely.

After the initial discovery of Dab1 and FE65 as LRP tail-binding proteins, extensive yeast two-hybrid screens were conducted using different parts of cytoplasmic tails from LDL receptor family members (16,
17). Several other gene products were identified that interact specifically with distinct regions in the cytoplasmic tails of LDL receptor family members. Some of these proteins also contain PTB domains (16-18), which are involved in receptor tail binding, whereas most of the others contain another type of protein interaction structure, PDZ domains. These newly identified adapter and scaffold proteins are key organizers or regulators of mitogen-activated protein (MAP) kinase signaling, ion channel function in the postsynaptic density, microtubular transport, nitric oxide signaling, and axon guidance. While specific functions of

Table 2

Adapter and scaffold proteins that bind to the intracellular domain

Disabled-1 (Dab1) Activation of Src family kinases, neuronal migration

FE65 APP processing?, actin remodeling

SEMCAP-1 Axon guidance, vesicular transport?

JIP1

JIP2

Regulation of MAPK and SAPK, including JNK

PSD-95

Scaffolding protein of the postsynaptic density, coupling to NMDA receptors

Talin-like protein Coupling to actin cytoskeleton

OMP25 Mitochondrial transport

CAPON Regulation of nitric oxide synthase

PIP4,5 kinase-like protein Regulation of inositol signaling

ICAP1 Integrin-mediated signaling?

She Ras activation 
LRP in most of these processes remain to be determined, it is now clear that LDL receptor family members are involved in at least some of them $(2,10,19)$.

\section{Lipoprotein metabolism}

When LRP was originally identified, its structural similarity to the LDL receptor and its expression in the liver suggested a role in lipoprotein metabolism and cholesterol homeostasis. Further in vitro evidence that LRP binds apoE led to the proposal that this molecule serves as a receptor for chylomicron remnants, lipoproteins that shuttle primarily dietary cholesterol from the gut to the liver. However, at the same time considerable evidence began to emerge suggesting that a major function of LRP lies in the removal of proteinase and proteinase inhibitor complexes (see below), raising the possibility that LRP may be a multifunctional scavenger receptor. A critical role of LRP, reaching beyond a restricted role in chylomicron remnant transport, was also suggested by gene knockout experiments that revealed an absolute requirement for LRP during the early stages of embryonic development in the mouse (20).

A role for LRP in lipoprotein metabolism has been strengthened by independent findings that revealed that LRP binds not only chylomicron remnants, but also the lipases that are directly involved in the generation of the remnant lipoproteins from triglyceride-rich chylomicrons (21). Several physiological and genetic studies in mice finally confirmed a function of LRP, in concert with the LDL receptor, in hepatic remnant metabolism (22-24). LRP is highly expressed not only in the liver, but also in virtually all neurons in the brain. Furthermore, astrocytes abundantly produce apoE in the CNS, raising the possibility that neuronal LRP mediates the neuronal uptake of astrocyte-derived cholesterol and other lipids. Such a function of LRP in the CNS is particularly intriguing in light of the role of apoE in the pathogenesis of late-onset Alzheimer disease and the role of cellular cholesterol content in the processing of APP to the amyloidogenic $A \beta$ peptide (25).

\section{LRP in proteinase metabolism}

Through its effects on certain serine proteinases and metalloproteinases, LRP serves as a crucial regulator of extracellular proteolytic activity. Urokinase-type plasminogen activator (uPA) and tissue-type plasminogen activator $(\mathrm{tPA})$ are serine proteinases that activate plasminogen and play important roles in wound repair, tumor invasion, and cell migration in both normal and pathologic situations (26). uPA is predominantly cellassociated, interacting with a cellular receptor, uPAR, and it initiates a proteolytic cascade that activates plasminogen to plasmin, which in turn can activate matrix metalloproteinases. This proteolytic pathway is regulated by a specific inhibitor, PA inhibitor-1 (PAI-1), which forms a stable complex with uPA. Once formed, this complex is rapidly internalized along with uPAR and is degraded (Figure 2) in a process mediated by LRP or other LDL receptor family members $(20,27)$. This degradative pathway regenerates unoccupied uPAR, which is necessary to sustain plasminogen activation on the cell surface. Failure to remove uPA:PAI- 1 complexes from the cell surface diminishes the capacity for plasminogen activation (28) and may impair the ability of uPA to mediate the removal of PAI-1 bound to vitronectin, thereby inhibiting uPA-dependent cellular migration (29). Finally, the interaction of uPA with uPAR initiates a variety of intracellular signaling events, such as calcium influx (30), formation of inositol-3phosphate (30), and activation of protein kinases such as the MAP kinase extracellular signal-related kinase (ERK) and its upstream regulator (31).

In addition to its effects on uPA and tPA levels and activity, LRP also regulates levels of certain matrix metalloproteinase (MMP) family members. Barmina et al. (32) demonstrated that the catabolism of MMP13 (collagenase-3) involves two receptors: a specific collagenase- 3 receptor that acts as a primary binding site on cells, and LRP, which is required for internalization of this enzyme. Yang et al. (33) found that antiLRP IgG and RAP can each inhibit adhesion and lead to increased MMP-2 levels in conditioned media from wild-type, but not thrombospondin-2-null, cells, confirming that clearance of MMP-2/thrombospondin-2 complexes is mediated by LRP. Finally, Hahn-Dantona et al. (34) demonstrated a direct high-affinity interaction between MMP-9 and LRP. The ability of LRP to modulate the levels of three MMPs (MMP-2, MMP-13, and MMP-9) indicates a major role for this receptor in removing excessive extracellular proteolytic activity. In the case of MMP-9, tight regulation of its activity and levels is important, since this MMP has roles in a variety of processes, including tumor progression and metastasis (35). LRP levels and activity are known to be substantially decreased in tumors (36), which would decrease the catabolism of MMP-9 and other proteinases, leading to higher levels of these enzymes at the tumor sites. Of interest in this regard, Kancha et al. (36) observed a correlation between invasive phenotype and low LRP expression in different subclones derived from a tumor cell line, although MMP-9 levels were not measured in these studies.

\section{Activation of lysosomal enzymes}

LRP is also indirectly involved in the regulation of the activity of lipid-degrading lysosomal enzymes that require a sphingolipid activator protein (SAP) for activation. Four distinct SAPs (A-D) are generated from a common SAP precursor protein by proteolytic cleavage in the lysosome. Genetic defects in the SAP precursor result in the lysosomal accumulation of several different types of cellular lipids due to enzymatic deficiencies in cerebrosidases, sphingomyelinases, glucosidases, and hexosaminidases (37). The SAP precursor is transported to the lysosome by two separate pathways involving an intracellular pathway by which SAP is shuttled directly from the trans-Golgi network to the lysosomes and an indirect pathway whereby SAP is first secreted by the cell and then internalized by the same or a neighboring cell. Two different receptor systems mediate SAP transport. Mannose-6-phosphate receptors are involved on one hand, and LRP on the other (38). Regulation of lysosomal lipid degradative pathways seems to be critical to a cell's survival, thus providing a ration- 
ale for a redundancy in SAP transport mechanisms. As a consequence, only minor accumulations of lysosomal lipids can be detected in LRP-deficient fibroblasts (S. Huettler et al., unpublished observations).

\section{Cellular entry of viruses and toxins}

In the course of evolution, LRP has been hijacked as an entry receptor for a family of viruses and for a bacterial toxin. Minor group rhinoviruses use members of the LDL receptor gene family, not exclusively LRP, as a docking receptor on the cell surface mediating their endocytic uptake into the target cell (39). Furthermore, a toxin from Pseudomonas aeruginosa, Pseudomonas exotoxin A, enters the cell exclusively via LRP (40). The latter is a single-chain polypeptide that harbors three distinct functional domains: a receptor-binding domain by which the toxin binds to LRP at the cell surface; a fusogenic domain, which mediates the translocation of the toxin into the cytoplasm; and an enzymatic domain that mediates ADP-ribosylation of elongation factor 2, thereby shutting down protein synthesis in the cell. Recently, a role for LRP in the activation of HIV has also been reported. In this case, LRP was shown to function as a receptor for the HIV-Tat protein, a transactivator for viral genes that is secreted by macrophages and other infected cells in which HIV replicates (41). The Tat protein binds LRP and thereby becomes internalized. It then leaves the endosomes by a process that is poorly understood and enters the nucleus of the target cells where it stimulates the transcription of dormant viral genes. This process is thought to be of particular importance in the nervous system where LRP is abundantly expressed on neurons and where it may accelerate the dementia that accompanies advanced HIV infection.

\section{Emerging functions in neurotransmission}

LRP present in neurons has the capacity to signal and may be involved in long-term potentiation (LTP) and in regulating synaptic plasticity. LTP is an electrophysiological parameter that is widely used to assess the strength and stability of synaptic connections. Such synaptic connectivity patterns are thought to be part of the structural basis for long-term memory. Studies in tPA-deficient mice (42) reveal that late-phase LTP is significantly impaired when compared with that in wildtype mice, and that it can be restored by adding exogenous tPA. The effect of tPA on LTP results from its association with LRP (43), as RAP inhibits tPA-mediated LTP. tPA has also been reported to potentiate NMDA receptor-mediated neurotoxicity via cleavage of the NR1 subunit of the NMDA receptor, leading to increased activity of the NMDA receptor (44). Another LRP ligand, $\alpha_{2} \mathrm{M}^{*}$, associates with neuronal LRP and induces a calcium influx via NMDA receptors (19), indicating a linkage between LRP and NMDA receptors, which could occur via the multivalent scaffold protein PSD-95 (Figure 2, right). PSD-95 associates with NMDA receptors as well as the cytoplasmic domain of LRP (16). The influx of calcium due to LRP-mediated activation of NMDA receptor channels is likely to affect a variety of downstream signaling cascades and may provide a mechanism of altering local synaptic plasticity.

\section{Potential roles in Alzheimer disease}

A number of findings suggest that LRP contributes to the pathobiology of Alzheimer disease (AD). LRP serves as a receptor for APP, apoE, and $\alpha_{2} \mathrm{M}$, all of which have been genetically linked to AD. Further, the levels of LRP decrease substantially with age (45), the major risk factor for nonfamilial AD. The contribution of LRP to AD is complex, and studies demonstrate that LRP has the capacity to influence both the production and the clearance of $A \beta$. The association of LRP with forms of APP that contain a Kunitz-type proteinase inhibitor (KPI) domain (46) alters APP processing, leading to increased $A \beta$ production (47). At the same time, the $A \beta$ peptide binds avidly to LRP ligands, such as $\alpha_{2} \mathrm{M}$ and apoE, and LRP-mediated clearance of these ligands complexed to $\mathrm{A} \beta$ contributes to a reduction in $\mathrm{A} \beta$ levels $(45,48)$. Interestingly, a silent polymorphism in exon 3 of the $L R P$ gene $(\mathrm{C} 776 \mathrm{~T})$ is associated with an altered risk for lateonset AD (49), and significantly lower levels of LRP in the brain have been reported in $\mathrm{AD}$ patients with the $\mathrm{C} / \mathrm{C}$ genotype compared with patients with the $\mathrm{C} / \mathrm{T}$ or $\mathrm{T} / \mathrm{T}$ genotype. Decreased LRP expression at clearance sites (perhaps at neurons or at sites along the capillary membranes) could lead to decreased $\alpha_{2} \mathrm{M}^{*}$ and/or apoEpromoted $A \beta$ catabolism, resulting in increased $A \beta$ deposition. At the same time, increased expression of LRP in activated glia in the AD brain is well documented and could lead to increased $A \beta$ production by these cells, also leading to increased $A \beta$ deposition. Finally, the signaling roles of LRP in response to ligand binding may be important for normal synaptic plasticity, and loss of LRP function or levels may impair these processes and lead to neuronal degeneration.

\section{Conclusion}

Our views of the functions of LRP, and of the LDL receptor gene family in general, are shifting. We can no longer think of this evolutionarily ancient and multifunctional receptor as a simple cargo transporter. The picture that is now emerging synthesizes the binding and removal of diverse biological ligands at the cell surface and the signals that many of these ligands evoke, informing the target cell of the conditions in its everchanging environment. Our goal now must be to understand the biochemical pathways along which these signals are transduced as well as the physiological functions they regulate.

\section{Acknowledgments}

J. Herz was an Established Investigator of the American Heart Association and Parke-Davis and is supported by grants from the NIH (HL-20948 and HL-63762), the Alzheimer Association, and the Perot Family Foundation. D.K. Strickland is supported by grants from the NIH (HL-50784, HL-65939, and HL-54710).

\footnotetext{
1. Willnow, T.E., Nykjaer, A., and Herz, J. 1999. Lipoprotein receptors: new roles for ancient proteins. Nat. Cell Biol. 1:E157-E162.

2. Herz, J., and Beffert, U. 2000. Apolipoprotein E receptors: linking brain development and Alzheimer disease. Nat. Rev. Neurosci. 1:51-58.

3. Goldstein, J.L., Hobbs, H.H., and Brown, M.S. 2001. Familial hypercholesterolemia. In Metabolic and molecular bases of inherited disease. C.R. Scriver et al., editors. McGraw-Hill Publishing Co. New York, New York, USA. $2863-2913$
} 
4. Neels, J.G., et al. 1999. The second and fourth cluster of class A cysteinerich repeats of the low density lipoprotein receptor-related protein share ligand-binding properties. J. Biol. Chem. 274:31305-13311.

5. Springer, T.A. 1998. An extracellular beta-propeller module predicted in lipoprotein and scavenger receptors, tyrosine kinases, epidermal growth factor precursor, and extracellular matrix components. J. Mol. Biol. 283:837-862.

6. Trommsdorff, M., Borg, J.P., Margolis, B., and Herz, J. 1998. Interaction of cytosolic adaptor proteins with neuronal apolipoprotein $\mathrm{E}$ receptors and the amyloid precursor protein. J. Biol. Chem. 273:33556-33560.

7. Huang, W., Dolmer, K., and Gettins, P.G. 1999. NMR solution structure of complement-like repeat CR8 from the low density lipoprotein receptor-related protein. J. Biol. Chem. 274:14130-14136.

8. Willnow, T.E., Orth, K., and Herz, J. 1994. Molecular dissection of ligand binding sites on the low density lipoprotein receptor-related protein. $J$. Biol. Chem. 269:15827-15832.

9. Chen, W.J., Goldstein, J.L., and Brown, M.S. 1990. NPXY, a sequence often found in cytoplasmic tails, is required for coated pit-mediated internalization of the low density lipoprotein receptor. J. Biol. Chem. 265:3116-3123.

10. Trommsdorff, M., et al. 1999. Reeler/Disabled-like disruption of neuronal migraton in knockout mice lacking the VLDL receptor and ApoE receptor-2. Cell. 97:689-701.

11. Bressler, S.L., et al. 1996. cDNA cloning and chromosome mapping of the human Fe65 gene: interaction of the conserved cytoplasmic domains of the human beta-amyloid precursor protein and its homologues with the mouse Fe65 protein. Hum. Mol. Genet. 5:1589-1598.

12. Zambrano, N., et al. 1997. Interaction of the phosphotyrosine interaction/phosphotyrosine binding-related domains of Fe 65 with wild-type and mutant Alzheimer's beta-amyloid precursor proteins. J. Biol. Chem. 272:6399-6405.

13. Ermekova, K.S., et al. 1997. The WW domain of neural protein FE65 interacts with proline-rich motifs in Mena, the mammalian homolog of Drosophila enabled. J. Biol. Chem. 272:32869-32877.

14. Guenette, S.Y., Chen, J., Jondro, P.D., and Tanzi, R.E. 1996. Association of a novel human FE65-like protein with the cytoplasmic domain of the betaamyloid precursor protein. Proc. Natl. Acad. Sci. USA. 93:10832-10837.

15. Duilio, A., Faraonio, R., Minopoli, G., Zambrano, N., and Russo, T. 1998. Fe65L2: a new member of the Fe65 protein family interacting with the intracellular domain of the Alzheimer's beta-amyloid precursor protein. Biochem. J. 330:513-519.

16. Gotthardt, M., et al. 2000. Interactions of the low density lipoprotein receptor gene family with cytosolic adaptor and scaffold proteins suggest diverse biological functions in cellular communication and signal transduction. J. Biol. Chem. 275:25616-25624.

17. Stockinger, W., et al. 2000. The reelin receptor ApoER2 recruits JNK-interacting proteins-1 and -2. J. Biol. Chem. 275:25625-25632.

18. Barnes, H., Larsen, B., Tyers, M., and van der Geer, P. 2001. Tyrosine-phosphorylated low density lipoprotein receptor-related protein 1 (LRP1) associates with the adaptor protein SHC in SRC-transformed cells. J. Biol. Chem. 276:19119-19125.

19. Bacskai, B.J., Xia, M.Q., Strickland, D.K., Rebeck, G.W., and Hyman, B.T. 2000. The endocytic receptor protein LRP also mediates neuronal calcium signaling via N-methyl-D-aspartate receptors. Proc. Natl. Acad. Sci. USA. 97:11551-11556.

20. Herz, J., Clouthier, D.E., and Hammer, R.E. 1992. LDL receptor-related protein internalizes and degrades uPA/PAI- 1 complexes and is essential for embryo implantation. Cell. 71:411-421.

21. Beisiegel, U., Weber, W., and Bengtsson-Olivecrona, G. 1991. Lipoprotein lipase enhances the binding of chylomicrons to low density lipoprotein receptor-related protein. Proc. Natl. Acad. Sci. USA. 88:8342-8346.

22. Willnow, T.E., Sheng, Z., Ishibashi, S., and Herz, J. 1994. Inhibition of hepatic chylomicron remnant uptake by gene transfer of a receptor antagonist. Science. 264:1471-1474.

23. Willnow, T.E., Armstrong, S.A., Hammer, R.E., and Herz, J. 1995. Functional expression of low density lipoprotein receptor-related protein is controlled by receptor-associated protein in vivo. Proc. Natl. Acad. Sci. USA. 92:4537-4541.

24. Rohlmann, A., Gotthardt, M., Hammer, R.E., and Herz, J. 1998. Inducible inactivation of hepatic LRP gene by cre-mediated recombination confirms role of LRP in clearance of chylomicron remnants. J. Clin. Invest. 101:689-695.

25. Simons, M., et al. 1998. Cholesterol depletion inhibits the generation of betaamyloid in hippocampal neurons. Proc. Natl. Acad. Sci. USA. 95:6460-6464.

26. Johnsen, M., Lund, L.R., Romer, J., Almholt, K., and Dano, K. 1998. Cancer invasion and tissue remodeling: common themes in proteolytic matrix degradation. Curr. Opin. Cell. Biol. 10:667-671.
27. Nykjaer, A., et al. 1992. Purified $\alpha_{2}$-macroglobulin receptor/LDL receptor-related protein binds urokinase:plasminogen activator inhibitor type1 complex. J. Biol. Chem. 267:14543-14546.

28. Zhang, J.C., et al. 1998. The low density lipoprotein receptor-related protein/alpha2- macroglobulin receptor regulates cell surface plasminogen activator activity on human trophoblast cells. J. Biol. Chem. 273:32273-32280

29. Stefansson, S., and Lawrence, D.A. 1996. The serpin PAI-1 inhibits cell migration by blocking integrin alpha $\mathrm{V}$ beta 3 binding to vitronectin. Nature. 383:441-443.

30. Sitrin, R.G., Pan, P.M., Harper, H.A., Blackwood, R.A., and Todd, R.F. 1999. Urokinase receptor (CD87) aggregation triggers phosphoinositide hydrolysis and intracellular calcium mobilization in mononuclear phagocytes. J. Immunol. 163:6193-200.

31. Nguyen, D.H., et al. 2000. Urokinase-type plasminogen activator stimulates the Ras/extracellular signal-regulated kinase (ERK) signaling pathway and MCF-7 cell migration by a mechanism that requires focal adhesion kinase, Src, and Shc. Rapid dissociation of GRB2/Sps-Shc complex is associated with the transient phosphorylation of ERK in urokinasetreated cells. J. Biol. Chem. 275:19382-19388.

32. Barmina, O.Y., et al. 1999. Collagenase-3 binds to a specific receptor and requires the low density lipoprotein receptor-related protein for internalization. J. Biol. Chem. 274:30087-30093.

33. Yang, Z., Strickland, D.K., and Bornstein, P. 2000. Extracellular MMP2 levels are regulated by the LRP scavenger receptor and thrombospondin 2. J. Biol. Chem. 106:12

34. Hahn-Dantona, E., Ruiz, J.F., Bornstein, P., and Strickland, D.K. 2001 The low-density-lipoprotein receptor-related protein modulates levels of matrix metalloproteinase 9 (MMP-9) by mediating its cellular catabolism. J. Biol. Chem. In press.

35. Bernhard, E.J., Hagner, B., Wong, C., Lubenski, I., and Muschel, R.J. 1995. The effect of E1A transfection on MMP-9 expression and metastatic potential. Int. J. Cancer. 60:718-724.

36. Kancha, R.K., Stearns, M.E., and Hussain, M.M. 1994. Decreased expression of the low density lipoprotein receptor-related protein/alpha 2macroglobulin receptor in invasive cell clones derived from human prostate and breast tumor cells. Oncol. Res. 6:365-372.

37. Sandhoff, K., Kolter, T., and Van Echten-Deckert, G. 1998. Sphingolipid metabolism. Sphingoid analogs, sphingolipid activator proteins, and the pathology of the cell. Ann. NY Acad. Sci. 845:139-151.

38. Hiesberger, T., et al. 1998. Cellular uptake of saposin (SAP) precursor and lysosomal delivery by the low density lipoprotein receptor-related protein (LRP). EMBO J. 17:4617-4625.

39. Hofer, F., et al. 1994. Members of the low density lipoprotein receptor family mediate cell entry of a minor-group common cold virus. Proc. Natl. Acad. Sci. USA. 91:1839-1842.

40. Kounnas, M.Z., et al. 1992 . The $\alpha_{2}$-macroglobulin receptor/low density lipoprotein receptor-related protein binds and internalizes Pseudomonas exotoxin A. J. Biol. Chem. 267:12420-12423.

41. Liu, Y., et al. 2000. Uptake of HIV-1 tat protein mediated by low-density lipoprotein receptor-related protein disrupts the neuronal metabolic balance of the receptor ligands. Nat. Med. 6:1380-1387.

42. Huang, Y.Y., et al. 1996. Mice lacking the gene encoding tissue-type plasminogen activator show a selective interference with late-phase long-term potentiation in both Schaffer collateral and mossy fiber pathways. Proc. Natl. Acad. Sci. USA. 93:8699-8704.

43. Zhuo, M., et al. 2000. Role of tissue plasminogen activator receptor LRP in hippocampal long- term potentiation. J. Neurosci. 20:542-549.

44. Nicole, O., et al. 2001. The proteolytic activity of tissue-plasminogen activator enhances NMDA receptor-mediated signaling. Nat. Med. 7:59-64.

45. Kang, D.E., et al. 2000. Modulation of amyloid beta-protein clearance and Alzheimer's disease susceptibility by the LDL receptor-related protein pathway. J. Clin. Invest. 106:1159-1166.

46. Kounnas, M.Z., et al. 1995. LDL receptor-related protein, a multifunctional ApoE receptor, binds secreted beta-amyloid precursor protein and mediates its degradation. Cell. 82:331-340.

47. Ulery, P.G., et al. 2000. Modulation of beta-amyloid precursor protein processing by the low density lipoprotein receptor-related protein (LRP). Evidence that LRP contributes to the pathogenesis of Alzheimer's disease. J. Biol. Chem. 275:7410-7415.

48. Shibata, M., et al. 2000. Clearance of Alzheimer's amyloid- $\beta(1-40)$ peptide from brain by LDL receptor-related protein-1 at the blood-brain barrier. J. Clin. Invest. 106:1489-1499.

49. Kang, D.E., et al. 1997. Genetic association of the low-density lipoprotein receptor-related protein gene (LRP), an apolipoprotein $\mathrm{E}$ receptor, with late-onset Alzheimer's disease. Neurology. 49:56-61. 